\title{
Spin excitation anisotropy as a probe of orbital ordering in the paramagnetic tetragonal phase of superconducting $\mathrm{BaFe}_{1.904} \mathrm{Ni}_{0.096} \mathbf{A s}_{2}$
}

\author{
Huiqian Luo, ${ }^{1}$ Meng Wang, ${ }^{1}$ Chenglin Zhang, ${ }^{2,3}$ Louis-Pierre Regnault, ${ }^{4}$ \\ Rui Zhang, ${ }^{1}$ Shiliang Li ${ }^{1}$ Jiangping $\mathrm{Hu},{ }^{1,5}$ and Pengcheng Dai ${ }^{2,3,1, *}$ \\ ${ }^{1}$ Beijing National Laboratory for Condensed Matter Physics, \\ Institute of Physics, Chinese Academy of Sciences, Beijing 100190, China \\ ${ }^{2}$ Department of Physics and Astronomy, Rice University, Houston, Texas 77005, USA \\ ${ }^{3}$ Department of Physics and Astronomy, The University of Tennessee, Knoxville, Tennessee 37996-1200, USA \\ ${ }^{4} S P S M S-M D N$, UMR-E CEA/UJF-Grenoble 1, INAC, Grenoble, F-38054, France \\ ${ }^{5}$ Department of Physics, Purdue University, West Lafayette, Indiana 47907, USA
}

(Dated: July 22, 2018)

\begin{abstract}
We use polarized neutron scattering to demonstrate that in-plane spin excitations in electron doped superconducting $\mathrm{BaFe}_{1.904} \mathrm{Ni}_{0.096} \mathrm{As}_{2}\left(T_{c}=19.8 \mathrm{~K}\right)$ change from isotropic to anisotropic in the tetragonal phase well above the antiferromagnetic (AF) ordering and tetragonal-to-orthorhombic lattice distortion temperatures $\left(T_{N} \approx T_{s}=33 \pm 2 \mathrm{~K}\right)$ without an uniaxial pressure. While the anisotropic spin excitations are not sensitive to the AF order and tetragonal-to-orthorhombic lattice distortion, superconductivity induces further anisotropy for spin excitations along the $[1,1,0]$ and $[1,-1,0]$ directions. These results indicate that the spin excitation anisotropy is a probe of the electronic anisotropy or orbital ordering in the tetragonal phase of iron pnictides.
\end{abstract}

PACS numbers: $74.70 . \mathrm{Xa}, 75.30 . \mathrm{Gw}, 78.70 . \mathrm{Nx}$

Understanding the electronic anisotropic state (electronic nematicity) at a temperature associated with the pseudogap phase is one of the most important unresolved problems in the quest for mechanism of high- $T_{c}$ superconductivity in copper oxides [1]. For iron pnictide superconductors derived from electron-doping to their antiferromagnetic (AF) parent compounds 2-4], there is considerable evidence for an anisotropic electronic state in the $\mathrm{AF}$ phase with an orthorhombic lattice distortion [5 7]. Upon warming to above the $\mathrm{AF}$ order $\left(T_{N}\right)$ and orthorhombic lattice distortion $\left(T_{s}\right)$ temperatures, iron pnictide superconductors become paramagnetic tetragonal metals [4]. Although transport [8], resonant ultrasound [9], angle-resolved photoemission spectroscopy (ARPES) [10], neutron scattering [11], and magnetic torque [12] measurements suggest an electronic anisotropy in the paramagnetic tetragonal phase, much is unclear about its microscopic origin. In one class of models, the observed electronic anisotropy in the paramagnetic tetragonal phase of iron pnictides [8 12$]$ may arise from either in-plane spin anisotropy (spin nematic phase) [13] as suggested from magnetic anisotropy in torque measurements [12], or orbital ordering [14 19] as implied from the energy splitting of the $d_{x z^{-}}$and $d_{y z^{-}}$ dominated bands above $T_{N}$ in ARPES [10]. However, there is no sufficient experimental evidence for spin nematic phase [20] and the observed orbital anisotropy in ARPES [10] may also be an extrinsic effect due to an uniaxial pressure induced increase in $T_{N}$ [21]. Instead of an electronic anisotropic spin nematic state or orbital ordering, the large resistivity anisotropy seen in electrondoped $\mathrm{BaFe}_{2-x} \mathrm{Co}_{x} \mathrm{As}_{2}$ [8] has been interpreted as due to anisotropic impurity scattering of Co-atoms in the FeAs layer [22, 23]. Since the in-plane resistivity anisotropy in charge transport property does not directly couple to spin and orbital order, these experimental results still leave open the question concerning the presence of spin nematicity or orbital ordering in the tetragonal phase of iron pnictides 13 19].

Here we use polarized neutron scattering to study the spin anisotropy in electron-doped iron pnictide superconductor $\mathrm{BaFe}_{1.904} \mathrm{Ni}_{0.096} \mathrm{As}_{2}\left(T_{c}=19.8 \mathrm{~K}\right)$ [24]. This material has incommensurate $\mathrm{AF}$ order $\left(T_{N}\right)$ and tetragonal-to-orthorhombic lattice distortion $\left(T_{s}\right)$ temperatures below $T_{N} \approx T_{s}=33 \pm 2 \mathrm{~K}$ (Fig. 1) [25]. Since the spin anisotropy in iron pnictide must originate from a spin-orbit coupling [26], its temperature dependence can provide direct information on any change of electronic physics involving spin or orbital degree of freedom. We demonstrate that spin excitations in $\mathrm{BaFe}_{1.904} \mathrm{Ni}_{0.096} \mathrm{As}_{2}$ exhibit an in-plane isotropic to anisotropic transition in the tetragonal phase at a temperature corresponding to the onset of in-plane resistivity anisotropy [8]. While the spin anisotropy shows no anomaly across $T_{N}$ and $T_{s}$, it enhances dramatically below $T_{c}$ revealing its connection to superconductivity. Since similar spin anisotropy is only observed in the AF orthorhombic phase of the undoped $\mathrm{BaFe}_{2} \mathrm{As}_{2}$ [27], spin-orbit coupling in the paramagnetic tetragonal phase of $\mathrm{BaFe}_{1.904} \mathrm{Ni}_{0.096} \mathrm{As}_{2}$ must be stabilized by an electronic anisotropic (nematic) phase or orbital ordering.

Figure 1(a) shows the schematic electronic phase diagram of $\mathrm{BaFe}_{2-x} \mathrm{Ni}_{x} \mathrm{As}_{2}$ as determined from neutron scattering 24] and transport measurements [28, 29]. In the tetragonal phase above the $T_{N}$ and $T_{s}$, transport measurements show anisotropic resistivity along 


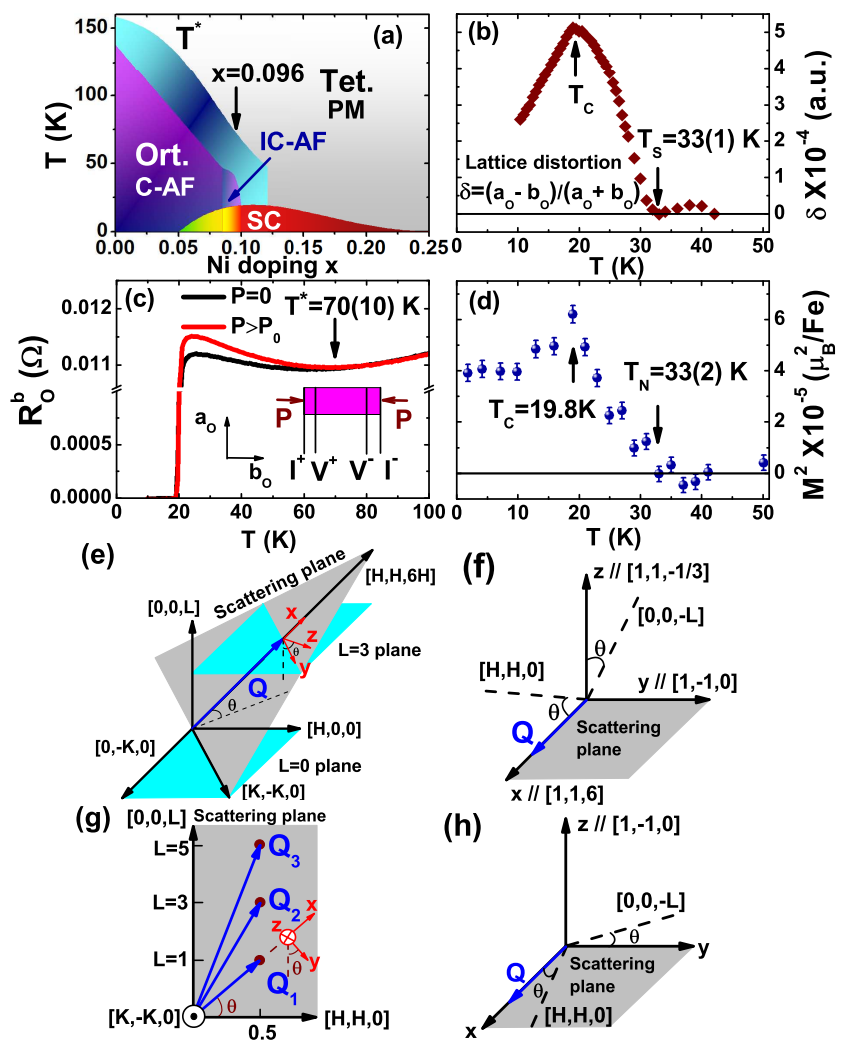

FIG. 1: (a)Electronic phase diagram of $\mathrm{BaFe}_{2-x} \mathrm{Ni}_{x} \mathrm{As}_{2}$ as a function of Ni doping $x$, where $T^{*}$ is the zone boundary of anisotropic in-plane resistivity obtained from ref. [29]. The arrow indicates the doping level $x=0.096$ for our experiments. (b) Orthorhombic lattice distortion order parameter $\delta$ shows $T_{s}=33 \pm 1 \mathrm{~K}$. The high resolution X-ray diffraction on nuclear peak $(2,2,12)$ experiment was from Ref. [25]. (c)In-plane resistance under zero and finite uniaxial stress $P$ along $b_{o}$, where $P=P_{0}$ is the detwinned pressure. From separate neutron scattering measurements, we know that $T_{N}$ and $T_{s}$ are uniaxial stress independent. (d) Temperature dependence of the AF order parameter shows $T_{N}=33 \pm 2 \mathrm{~K}$. $(\mathrm{e}, \mathrm{g})$ Scattering plane and neutron polarization directions in our experiments. (f,h) Magnetic response of SF channels in the neutron polarization analysis.

the orthorhombic $a_{o} / b_{o}$ directions below the electronic nematic ordering temperature $T^{*}$ [8]. We chose to study $\mathrm{BaFe}_{1.904} \mathrm{Ni}_{0.096} \mathrm{As}_{2}$ because this sample has coexisting short-range incommensurate $\mathrm{AF}$ order and superconductivity 24]. From previous high-resolution Xray diffraction experiments on $\mathrm{BaFe}_{2-x} \mathrm{Co}_{x} \mathrm{As}_{2}$ [30] and $\mathrm{BaFe}_{2-x} \mathrm{Ni}_{x} \mathrm{As}_{2}$ [25], we know that $\mathrm{BaFe}_{1.904} \mathrm{Ni}_{0.096} \mathrm{As}_{2}$ changes from tetragonal to orthorhombic lattice structure below $T_{s}$, and the lattice orthorhombicity becomes smaller on entering the superconducting state. Figure 1(b) shows the temperature dependence of orthorhombicity $\delta=\left(a_{o}-b_{o}\right) /\left(a_{o}+b_{o}\right)$, revealing $T_{s}=33 \pm 1 \mathrm{~K}$ 25]. Although the orthorhombicity of the system clearly decreases on cooling below $T_{c}$, its lattice structure does not become fully tetragonal at $10 \mathrm{~K}$ [Fig. 1(b)]. Simi-

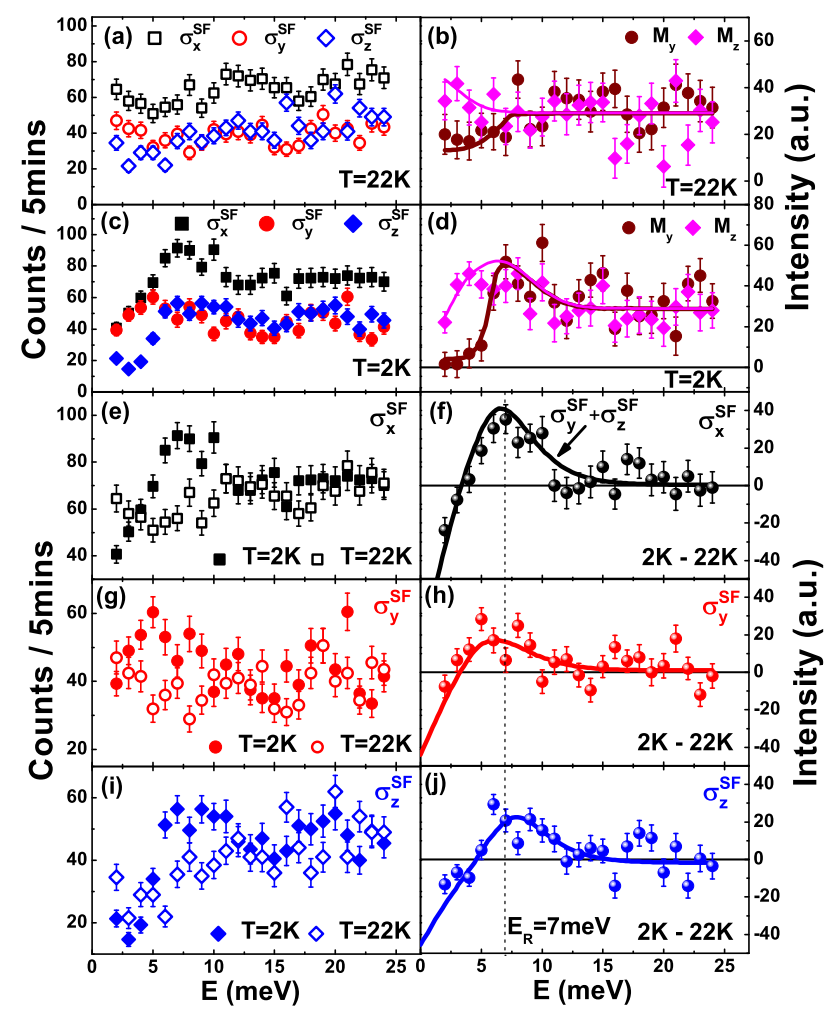

FIG. 2: (a) Energy scans at $\mathbf{Q}=(0.5,0.5,3)$ for SF scattering at $22 \mathrm{~K}$ above $T_{c}$ for different neutron polarization directions, marked as $\sigma_{x, y, z}^{\mathrm{SF}}$. (b) The magnetic response $M_{y}$ and $M_{z}$ extracted from (a). (c) and (d) Identical energy scans at $2 \mathrm{~K}$ below $T_{c}$ in the neutron SF channel and $M_{y}, M_{z}$, respectively. (e) The total neutron SF scattering $\sigma_{x}^{\mathrm{SF}}$ at $2 \mathrm{~K}$ and $22 \mathrm{~K}$ and (f) their difference, where a neutron spin resonance is seen at $E_{r}=7 \mathrm{meV}$. (g) The $\sigma_{y}^{\mathrm{SF}}$ at $2 \mathrm{~K}$ and $22 \mathrm{~K}$ and $(\mathrm{h})$ their difference. ( $\mathrm{i}, \mathrm{j}$ ) Identical scans for $\sigma_{z}^{\mathrm{SF}}$. The solid lines in (b, $\mathrm{d}, \mathrm{h}, \mathrm{j}$ ) are guides to the eyes, and in (f) the solid line is the sum of $(\mathrm{h})$ and $(\mathrm{j})$.

larly, temperature dependence of the magnetic order parameter indicates a Néel temperature of $T_{N}=33 \pm 2 \mathrm{~K}$ [Fig. 1(d)] 24]. To confirm the anisotropic resistivity in the tetragonal phase of $\mathrm{BaFe}_{1.904} \mathrm{Ni}_{0.096} \mathrm{As}_{2}$, we have also carried out resistivity measurements on a detwinned sample. The outcome shows clear resistivity anisotropy for temperatures below $T^{*}=70 \pm 10 \mathrm{~K}$ [Fig. 1(c)].

We prepared sizable high quality single crystals of $\mathrm{BaFe}_{1.904} \mathrm{Ni}_{0.096} \mathrm{As}_{2}$ using self-flux method 28] and coaligned $\sim 11 \mathrm{~g}$ single crystals within $3^{\circ}$ full width at half maximum (FWHM). Our polarized neutron scattering experiments were carried out using IN22 thermal triple-axis spectrometer at the Institut Laue-Langevin, Grenoble, France [26]. The scattering planes are $[H, H, 6 H] \times[K,-K, 0]$ and $[H, H, 0] \times[0,0, L]$ to probe the wave vector dependence of spin excitations along different directions. Using pseudo-tetragonal lattice unit cell with $a \approx b \approx 3.956 \AA$, and $c=12.92 \AA$, the vector $\mathbf{Q}$ in three-dimensional reciprocal space in $\AA^{-1}$ is defined 
as $\mathbf{Q}=H \mathbf{a}^{*}+K \mathbf{b}^{*}+L \mathbf{c}^{*}$, where $H, K$, and $L$ are Miller indices and $\mathbf{a}^{*}=\hat{\mathbf{a}} 2 \pi / a, \mathbf{b}^{*}=\hat{\mathbf{b}} 2 \pi / b, \mathbf{c}^{*}=\hat{\mathbf{c}} 2 \pi / c$ are reciprocal lattice units. We define neutron polarization directions as $x, y, z$, with $x$ parallel to $\mathbf{Q}, y$ and $z$ perpendicular to $\mathbf{Q}$ as shown in Figs. 1(e) and 1(g). At the $\mathrm{AF}$ wave vector $\mathbf{Q}=(0.5,0.5,3)$, neutron polarization directions $x$ and $y$ are parallel to the $\mathbf{Q}=[1,1,6]$ and $[1,-1,0]$ respectively, while $z$ is perpendicular to the $[H, H, 6 H] \times[K,-K, 0]$ scattering plane along the $\mathbf{Q}=[1,1,-1 / 3]$ direction [Figs. 1(e) and 1(f)]. In the $[H, H, L]$ scattering plane, we probe $\mathrm{AF}$ wave vectors $\mathbf{Q}_{\mathbf{1}}=(0.5,0.5,1), \mathbf{Q}_{\mathbf{2}}=(0.5,0.5,3), \mathbf{Q}_{\mathbf{3}}=(0.5,0.5,5)$, where neutron polarization directions $x, y$, and $z$ are shown in Fig. 1(g).

Since neutron scattering is only sensitive to magnetic scattering component perpendicular to the momentum transfer $\mathbf{Q}$, magnetic responses within the $y-z$ plane $\left(M_{y}\right.$ and $\left.M_{z}\right)$ can be measured by using different neutron spin directions [Figs. 1(f) and 1(h)]. At a specific momentum and energy transfer, scattered neutrons can have polarizations antiparallel (neutron spin flip or SF, $\uparrow \downarrow$ ) to the incident neutrons. Therefore, the three neutron SF scattering cross sections can be written as $\sigma_{\alpha}^{\mathrm{SF}}$, where $\alpha=x, y, z$. The magnetic moments $M_{y}$ and $M_{z}$ can be extracted via $\sigma_{x}^{\mathrm{SF}}-\sigma_{y}^{\mathrm{SF}}=c M_{y}$ and $\sigma_{x}^{\mathrm{SF}}-\sigma_{z}^{\mathrm{SF}}=c M_{z}$, where $c=(R-1) /(R+1)$ and the flipping ratio $R$ is measured by the leakage of NSF nuclear Bragg peaks into the magnetic SF channel $R=\sigma_{\text {Bragg }}^{\mathrm{NSF}} / \sigma_{\text {Bragg }}^{\mathrm{SF}} \approx 15[26]$.

In previous polarized neutron scattering experiments on optimally electron-doped iron pnictide superconductor $\mathrm{BaFe}_{1.9} \mathrm{Ni}_{0.1} \mathrm{As}_{2}$ [26] and $\mathrm{BaFe}_{1.88} \mathrm{Co}_{0.12} \mathrm{As}_{2}$ [31] without static AF order, low-energy spin excitations were found to be anisotropic in the superconducting state. For electron-overdoped $\mathrm{BaFe}_{1.85} \mathrm{Ni}_{0.15} \mathrm{As}_{2}$, spin excitations are isotropic in both the normal and superconducting states 32]. Figure 2(a) shows energy scans at $\mathbf{Q}=(0.5,0.5,3)$ for all three SF channels $\left(\sigma_{\alpha}^{\mathrm{SF}}\right)$ at $T=22$ $\mathrm{K}$. For a pure isotropic paramagnetic scattering, one expects $\sigma_{x}^{\mathrm{SF}}=2 \sigma_{y}^{\mathrm{SF}}=2 \sigma_{z}^{\mathrm{SF}}$ assuming a small (negligible) background scattering [26, 31]. While this is indeed the case for $E \geq 5 \mathrm{meV}$, there is apparent spin anisotropy for $E<5 \mathrm{meV}$ with $\sigma_{y}^{\mathrm{SF}}>\sigma_{x}^{\mathrm{SF}} / 2>\sigma_{z}^{\mathrm{SF}}$ [Fig. 2(a)]. On cooling to $T=2 \mathrm{~K}$, the spectra are re-arranged [Fig. 2(c)]. While there is a clear resonance at $E_{r} \approx 7 \mathrm{meV}$ in the $\sigma_{x}^{\mathrm{SF}}$ channel at the expense of lower energy spin excitations [Figs. 2(e)], $\sigma_{y}^{\mathrm{SF}}$ and $\sigma_{z}^{\mathrm{SF}}$ respond to superconductivity very differently. Instead of showing suppressed spin fluctuations below $4 \mathrm{meV}$ as in the temperature difference plot for $\sigma_{x}^{\mathrm{SF}}$, superconductivity induces a very broad resonance in $\sigma_{y}^{\mathrm{SF}}$ with magnetic intensity gain from 3 to $10 \mathrm{meV}$ [Figs. $2(\mathrm{~g})$ and $2(\mathrm{~h})$ ]. This is similar to the $c$-axis polarized spin excitations of $\mathrm{BaFe}_{1.9} \mathrm{Ni}_{0.1} \mathrm{As}_{2}$ below $T_{c}$ [26] and $\mathrm{BaFe}_{1.88} \mathrm{Co}_{0.12} \mathrm{As}_{2}$ [31]. For $\sigma_{z}^{\mathrm{SF}}$, the effect of superconductivity is to open a larger spin gap below about $5 \mathrm{meV}$ and form a resonance near $E_{r}=7$ meV [Figs. 2(i) and 2(j)]. Since the temperature differ-

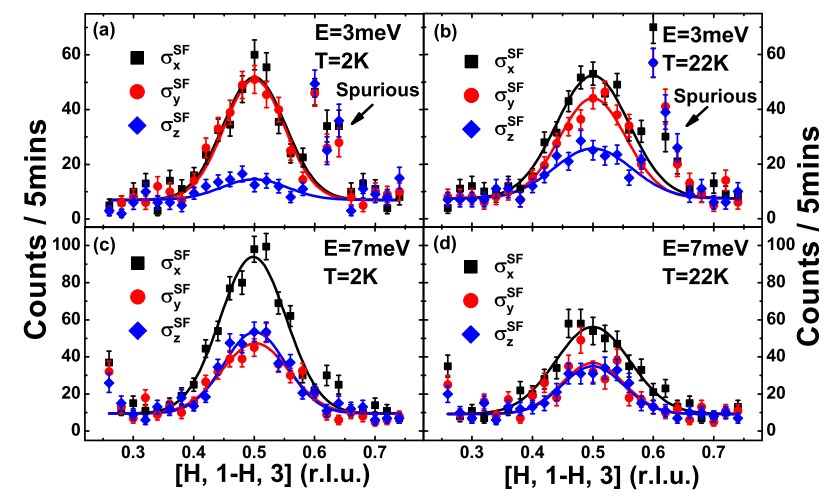

FIG. 3: (a) and (b) Constant energy scans at $3 \mathrm{meV}$ in the neutron SF channel $\sigma_{x, y, z}^{\mathrm{SF}}$ below and above $T_{c}$, respectively. The solid lines are Gaussian fits to the data on linear backgrounds. (c) and (d) Identical scans and results at the resonance energy of $E_{r}=7 \mathrm{meV}$.

ence plots in Figs. 2(f), 2(h), and 2(j) should contain no background, we expect $\sigma_{x}^{\mathrm{SF}}=\sigma_{y}^{\mathrm{SF}}+\sigma_{z}^{\mathrm{SF}}$. The solid line in Fig. 2(f) shows the sum of $\sigma_{y}^{\mathrm{SF}}$ and $\sigma_{z}^{\mathrm{SF}}$, and it is indeed statistically identical to $\sigma_{x}^{\mathrm{SF}}$.

To quantitatively estimate the spin anisotropy from $\sigma_{\alpha}^{\mathrm{SF}}$ in Figs. 2(a) and 2(c), we plot in Figs. 2(b) and 2(d) the energy dependence of $M_{y}$ and $M_{z}$ in the normal and superconducting states, respectively. At $T=22 \mathrm{~K}$, the magnetic scattering show spin anisotropy below $\sim 5$ $\mathrm{meV}$. At $2 \mathrm{~K}$, the $M_{y}$ shows a clean spin gap below 4 meV and a resonance at $E_{r}=7 \mathrm{meV}$, while $M_{z}$ shows a broad peak centered around $5 \mathrm{meV}$. In previous polarized neutron scattering experiments on electron-doped iron pnictide superconductors [26, 31], similar magnetic anisotropy was found at low-energies.

Figure 3 summarizes constant energy scans along the $[H, 1-H, 3]$ direction at $E=3$ and $7 \mathrm{meV}$ with different neutron polarizations. At $T=2 \mathrm{~K}, \sigma_{x}^{\mathrm{SF}}$ and $\sigma_{y}^{\mathrm{SF}}$ at $E=3 \mathrm{meV}$ display well-defined peaks at $(0.5,0.5,3)$ with almost the same magnitude, while $\sigma_{z}^{\mathrm{SF}}$ has only a broad weak peak center at at $(0.5,0.5,3)$ [Fig. $3(\mathrm{a})]$. These data are consistent with constant- $Q$ scans in Fig. 2. At $T=$ $22 \mathrm{~K}$, similar scans show three separate peaks satisfying $\sigma_{y}^{\mathrm{SF}}>\sigma_{x}^{\mathrm{SF}} / 2>\sigma_{z}^{\mathrm{SF}}$, again confirming the anisotropic nature of the normal state spin excitations in Fig. 2(a). For comparison, spin excitations at the resonance energy of $E_{r}=7 \mathrm{meV}$ are completely isotropic below [Fig. 3(c)] and above [Fig. 3(d)] $T_{c}$ satisfying $\sigma_{x}^{\mathrm{SF}}=2 \sigma_{y}^{\mathrm{SF}}=2 \sigma_{z}^{\mathrm{SF}}$.

Given the clear experimental evidence for anisotropic spin excitations at $E=3 \mathrm{meV}$ and its possible coupling to superconductivity as illustrated in Figs. 2 and 3, it would be interesting to measure the temperature dependence of the spin anisotropy. Figure 4(a) shows the temperature dependent scattering for $\sigma_{\alpha}^{\mathrm{SF}}$ at $\mathbf{Q}=(0.5,0.5,3)$ and $E=3 \mathrm{meV}$. At temperatures above $70 \mathrm{~K}$, we see $\sigma_{x}^{\mathrm{SF}} \approx 2 \sigma_{y}^{\mathrm{SF}} \approx 2 \sigma_{z}^{\mathrm{SF}}$ indicating that spin excitations are isotropic with $M_{y}=M_{z}$. On cooling to below $70 \mathrm{~K}$, we 


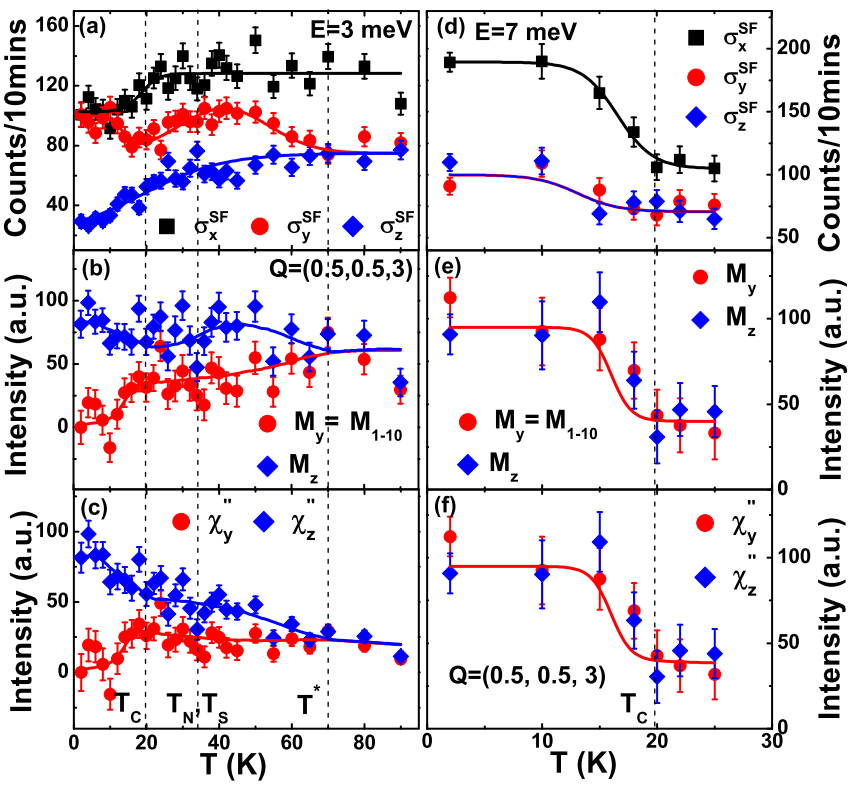

FIG. 4: (a) Temperature dependence of neutron SF scattering cross section $\sigma_{x, y, z}^{\mathrm{SF}}$ at $3 \mathrm{meV}$ and $\mathbf{Q}=(0.5,0.5,3)$. (b) The temperature dependence of magnetic response along the $[1,-1,0]\left(M_{y}\right)$ and $[1,1,-1 / 3]\left(M_{z}\right)$ directions. Clear anisotropy persists up to $T^{*}=70 \mathrm{~K}$. (c) Temperature dependence of the dynamic susceptibility, $\chi_{y}^{\prime \prime}$ and $\chi_{z}^{\prime \prime}$. (d),(e), and (f) Identical results at the resonance energy of $E_{r}=7 \mathrm{meV}$.

see a clear splitting of the temperature dependent $\sigma_{y}^{\mathrm{SF}}$ and $\sigma_{z}^{\mathrm{SF}}$. While $\sigma_{x}^{\mathrm{SF}}$ shows no visible changes cross 70 $\mathrm{K}, \sigma_{y}^{\mathrm{SF}}$ increases and $\sigma_{z}^{\mathrm{SF}}$ decreases with decreasing temperature below $70 \mathrm{~K}$ before saturating around $40 \mathrm{~K}$. On cooling further to crossing $T_{N}$ and $T_{s}$, there are no statistically significant changes in $\sigma_{x}^{\mathrm{SF}}, \sigma_{y}^{\mathrm{SF}}$, or $\sigma_{z}^{\mathrm{SF}}$, indicating that spin anisotropy at $E=3 \mathrm{meV}$ does not respond to $\mathrm{AF}$ ordering and tetragonal-orthorhombic lattice distortion. Finally, on cooling below $T_{c}$, we see a clear reduction in $\sigma_{x}^{\mathrm{SF}}$, revealing a suppression of the spin excitations for energies below the resonance. On the other hand, while $\sigma_{y}^{\mathrm{SF}}$ increases at $T_{c}$ and merges with $\sigma_{x}^{\mathrm{SF}}$ below around $10 \mathrm{~K}, \sigma_{z}^{\mathrm{SF}}$ exhibits a further reduction in intensity below $T_{c}$. Figure 4(b) shows the temperature dependence of the magnetic scattering $M_{y}$ and $M_{z}$ obtained from $\sigma_{\alpha}^{\mathrm{SF}}$. On cooling, spin excitations first change from isotropic to anisotropic below approximately $70 \mathrm{~K}$, and further enhance anisotropy below $T_{c}$ with almost zero $M_{y}$ at $2 \mathrm{~K}$. Figure 4(c) shows temperature dependence of the imaginary part of the dynamic susceptibility, $\chi^{\prime \prime}$, along the $y$ and $z$ directions. They show again the appearance of spin anisotropy below $70 \mathrm{~K}$ with no changes across $T_{N}$ and $T_{s}$, and a further spin anisotropy change below $T_{c}$.

Figure 4(d) shows temperature dependence of the magnetic intensity at the resonance energy $E_{r}=7 \mathrm{meV}$. At all measured temperatures, we find $\sigma_{x}^{\mathrm{SF}} \approx 2 \sigma_{y}^{\mathrm{SF}} \approx 2 \sigma_{z}^{\mathrm{SF}}$, thus confirming the isotropic nature of the mode. Figures

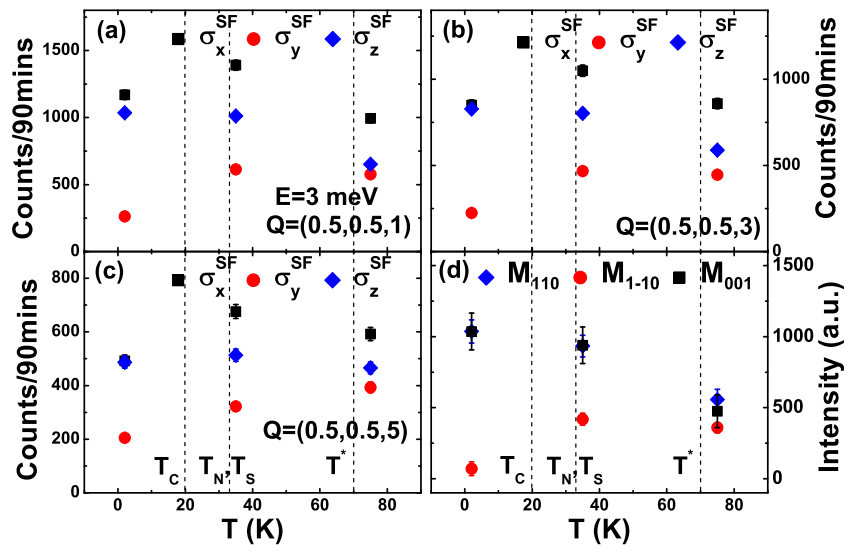

FIG. 5: (a,b,c) Temperature dependence of neutron SF scattering cross section $\sigma_{x, y, z}^{\mathrm{SF}}$ at $3 \mathrm{meV}$ and $\mathbf{Q}_{\mathbf{1}, \mathbf{2}, \mathbf{3}}=(0.5,0.5, L)$ with $L=1,3,5$. (d) The temperature dependence of magnetic response along the $[1,1,0]\left(M_{110}\right),[1,-1,0]\left(M_{1 \overline{1} 0}\right)$, and $[0,0,1]\left(M_{001}\right)$ directions. Clear in-plane anisotropy persists up to $T^{*}=70 \mathrm{~K}$.

4(e) and 4(f) are the corresponding temperature dependence of $M_{y}, M_{z}$ and $\chi_{y}^{\prime \prime}, \chi_{z}^{\prime \prime}$, respectively. In both cases, there is intensity increase below $T_{c}$, consistent with earlier work on the resonance [26, 31]. For comparison, we note that spin excitations in superconducting iron chalcogenides have slightly anisotropic resonance with isotropic spin excitations below it [33, 34].

In previous polarized neutron measurements on the parent compound $\mathrm{BaFe}_{2} \mathrm{As}_{2}$ [27], it was found that the in-plane polarized spin waves exhibit a larger gap than the out-of-plane polarized ones, suggesting that it costs more energy to rotate a spin within the orthorhombic $a-b$ plane than to rotate it perpendicular to the FeAs layers. However, the spin anisotropy immediately disappears in the paramagnetic tetragonal state above $T_{N}$ and $T_{s}$ [27]. Since $M_{y}$ is the spin moment in the FeAs layers [Fig. 1(e)], the $M_{y}$ and $M_{z}$ anisotropy should also represent the spin anisotropy along the $[1,-1,0]$ and $[1,1,-1 / 3]$ directions, respectively. To determine the precise anisotropic direction of spin excitations at $E=3 \mathrm{meV}$, we measured $\sigma_{\alpha}^{\mathrm{SF}}$ at $\mathbf{Q}_{\mathbf{1 , 2}, \mathbf{3}}$ in the $[H, H, L]$ zone [Figs. $5(\mathrm{a})-5(\mathrm{c})]$. At $T=2 \mathrm{~K}\left(\ll T_{c}\right)$, we see $\sigma_{x}^{\mathrm{SF}} \approx \sigma_{z}^{\mathrm{SF}} \gg \sigma_{y}^{\mathrm{SF}}$ at all wave vectors probed. On warming to $35 \mathrm{~K}\left(>T_{N}, T_{s}\right)$, we have $\sigma_{x}^{\mathrm{SF}}>\sigma_{z}^{\mathrm{SF}}>\sigma_{y}^{\mathrm{SF}}$. At $75 \mathrm{~K}$, we find $\sigma_{x}^{\mathrm{SF}} \approx 2 \sigma_{y}^{\mathrm{SF}} \approx 2 \sigma_{z}^{\mathrm{SF}}$, suggesting weak or no spin anisotropy. By considering wave vector dependence of spin excitations in Figs. 5(a)-5(c), we estimate the temperature dependence of $M_{110}, M_{1 \overline{1} 0}$, and $M_{001}$ [Fig. $5(\mathrm{~d})]$ [35].

In the superconducting orthorhombic state, there are clear in-plane magnetic anisotropy with $M_{001} \sim M_{110} \gg$ $M_{1 \overline{1} 0} \approx 0$. In the paramagnetic tetragonal state just above $T_{s}$ and $T_{N}$, we still have strong in-plane magnetic anisotropy with $M_{110} \sim M_{001}>M_{1 \overline{1} 0}$. This is surprising 
because domains associated with the in-plane $\mathrm{AF}$ wave vector $\mathbf{Q}=(0.5,0.5)$ are randomly mixed with those associated with the $\mathbf{Q}=(0.5,-0.5)$ in the tetragonal phase. In the AF orthorhombic state, the low-energy spin excitations associated with the $\mathbf{Q}=(0.5,0.5)$ domains are well separated from those associated with $\mathbf{Q}=(0.5,-0.5)$ in reciprocal space [11]. If there are strong paramagnetic scattering at $\mathbf{Q}=(0.5,-0.5)$ arising from domains associated with $\mathbf{Q}=(0.5,0.5)$ in the tetragonal phase, one should not be able to determine the spin excitation anisotropy in neutron polarization analysis. However, recent unpolarized neutron experiments on nearly $100 \%$ mechanically detwinned $\mathrm{BaFe}_{2-x} \mathrm{Ni}_{x} \mathrm{As}_{2}$ reveal that spin excitations in the paramagnetic tetragonal state are still centered mostly at $\mathbf{Q}=(0.5,0.5)$ 36]. Therefore, our neutron polarization analysis provides the most compelling evidence for the in-plane spin anisotropy in the paramagnetic tetragonal phase of $\mathrm{BaFe}_{1.904} \mathrm{Ni}_{0.096} \mathrm{As}_{2}$ [Fig. 5(d)]. Since such spin excitation anisotropy occurs at the $\mathrm{AF}$ wave vector $\mathbf{Q}=(0.5,0.5)$, it does not break the $C_{4}$ rotational symmetry of the underlying lattice.

In summary, we have discovered that an in-plane isotropic to anisotropic spin fluctuation transition occurs in the tetragonal phase of superconducting $\mathrm{BaFe}_{1.904} \mathrm{Ni}_{0.096} \mathrm{As}_{2}$ without an uniaxial pressure, consistent with resistivity anisotropy. The spin anisotropy is further enhanced upon entering into the superconducting state. Therefore, our experimental results establish the in-plane spin anisotropy as a new experimental probe to study the spontaneously broken electronic symmetries in strain free iron pnictides.

The work at IOP, CAS, is supported by MOST (973 project: 2012CB821400, 2011CBA00110, and 2010CB833102) and NSFC (No.11004233). The work at UTK and Rice is supported by the U.S. NSF-DMR1063866.

* Electronic address: pdai@rice.edu

[1] E. Fradkin, S. A. Kivelson, M. J. Lawler, J. P. Eisenstein, and A. P. Mackenzie, Annu. Rev. Condens. Matter Phys. 1, 153 (2010).

[2] Y. Kamihara, T. Watanabe, M. Hirano, and H. Hosono, J. Am. Chem. Soc. 130, 3296-3297 (2008).

[3] C. de la Cruz et al., Nature (London) 453,899 (2008).

[4] P. Dai, J. P. Hu, and E. Dagotto, Nature Phys. 8, 709 (2012).

[5] J. Zhao, D. T. Adroja, D. X. Yao, R. Bewley, S. Li, X. F. Wang, G. Wu, X. H. Chen, J. P. Hu, and P. C. Dai, Nature Phys. 5, 555 (2009).

[6] T.-M. Chuang, M. P. Allan, Jinho Lee, Yang Xie, Ni Ni, S. L. Bud'ko, G. S. Boebinger, P. C. Canfield, J. C. Davis, Science 327, 181 (2010).

[7] T. Shimojima et al., Phys. Rev. Lett. 104, 057002 (2010).

[8] I. R. Fisher, L. Degiorgi, and Z. X. Shen, Rep. Prog. Phys. 74, 124506 (2011).
[9] R. M. Fernandes, L. H. VanBebber, S. Bhattacharya, P. Chandra, V. Keppens, D. Mandrus, M. A. McGuire, B. C. Sales, A. S. Sefat, and J. Schmalian, Phys. Rev. Lett. 105, 157003 (2010).

[10] M. Yi et al., Proc. Natl. Acad. Sci. U.S.A. 108, 6878 (2011).

[11] L. W. Harriger, H. Q. Luo, M. S. Liu, C. Frost, J. P. Hu, M. R. Norman, and P. C. Dai, Phys. Rev. B 84, 054544 (2011).

[12] S. Kasahara, H. J. Shi, K. Hashimoto, S. Tonegawa, Y. Mizukami, T. Shibauchi, K. Sugimoto, T. Fukuda, T. Terashima, Andriy H. Nevidomskyy, and Y. Matsuda, Nature 486, 382 (2012).

[13] J. P. Hu and C. K. Xu, Physica C 481, 215 (2012).

[14] C. C. Lee, W. G. Yin, and W. Ku, Phys. Rev. Lett. 103, 267001 (2009).

[15] F. Krüger, S. Kumar, J. Zaanen, and J. van den Brink, Phys. Rev. B 79, 054504 (2009).

[16] W. C. Lv, J. S. Wu, and P. Phillips, Phys. Rev. B 80, 224506 (2009).

[17] M. Daghofer, Q.-L. Luo, R. Yu, D. X. Dao, A. Moreo, and E. Dagotto, Phys. Rev. B 81, 180514(R) (2010).

[18] C.-C. Chen, J. Maciejko, A. P. Sorini, B. Moritz, R. R. P. Singh, and T. P. Devereaux, Phys. Rev. B 82, 100504(R) (2010).

[19] B. Valenzuela, E. Bascones, and M. J. Calderón, Phys. Rev. Lett. 105, 207202 (2010).

[20] M. Nakajima, S. Ishida, Y. Tomioka, K. Kihou, C. H. Lee, A. Iyo, T. Ito, T. Kakeshita, H. Eisaki, and S. Uchida, Phys. Rev. Lett. 109, 217003 (2012).

[21] C. Dhital, Z. Yamani, W. Tian, J. Zeretsky, A. S. Sefat, Z. Wang, R. J. Birgeneau, and S. D. Wilson, Phys. Rev. Lett. 108, 087001 (2012).

[22] S. Ishida, M. Nakajima, T. Liang, K. Kihou, C. H. Lee, A. Iyo, H. Eisaki, T. Kakeshita, Y. Tomioka, T. Ito, and S. Uchida, Phys. Rev. Lett. 110, 207001 (2013).

[23] M. P. Allan, T.-M. Chuang, F. Massee, Yang Xie, Ni Ni, S. L. Bud'ko, G. S. Boebinger, Q. Wang, D. S. Dessau, P. C. Canfield, M. S. Golden, J. C. Davis, Nature Phys. 9, 220 (2013).

[24] H. Q. Luo, R. Zhang, M. Laver, Z. Yamani, M. Wang, X. Y. Lu, M. Y. Wang, Y. C. Chen, S. Li, S. Chang, J. W. Lynn, and P. C. Dai, Phys. Rev. Lett. 108, 247002 (2012).

[25] X. Y. Lu, H. Gretarsson, R. Zhang, X. R. Liu, H. Q. Luo, W. Tian, M. Laver, Z. Yamani, Y.-J. Kim, A. H. Nevidomskyy, Q. Si, and P. C. Dai, Phys. Rev. Lett. 110, 257001 (2013).

[26] O. J. Lipscombe, L. W. Harriger, P. G. Freeman, M. Enderle, C. L. Zhang, M. Y. Wang, T. Egami, J. P. Hu, T. Xiang, M. R. Norman, and P. C. Dai, Phys. Rev. B 82, 064515 (2010).

[27] N. Qureshi, P. Steffens, S. Wurmehl, S. Aswartham, B. Büchner, and M. Braden, Phys. Rev. B 86, 060410(R) (2012).

[28] Y. C. Chen et al., Supercond. Sci. Technol. 24, 065004 (2011).

[29] H. -H. Kuo, J.-H. Chu, S. C. Riggs, L. Yu, P. L. McMahon, K. De Greve, Y. Yamamoto, J. G. Analytis, and I. R. Fisher, Phys. Rev. B 84, 054540 (2011).

[30] S. Nandi, M. G. Kim, A. Kreyssig, R. M. Fernandes, D. K. Pratt, A. Thaler, N. Ni, S. L. Bud'ko, P. C. Canfield, J. Schmalian, R. J. McQueeney, and A. I. Goldman, Phys. Rev. Lett. 104, 057006 (2010). 
[31] P. Steffens, C. H. Lee, N. Qureshi, K. Kihou, A. Iyo, H. Eisaki, and M. Braden, Phys. Rev. Lett. 110, 137001 (2013).

[32] M. S. Liu, C. Lester, J. Kulda, X. Y. Lu, H. Q. Luo, M. Wang, S. M. Hayden, and P. C. Dai, Phys. Rev. B 85, 214516 (2012).

[33] P. Babkevich, B. Roessli, S. N. Gvasaliya, L.-P. Regnault, P. G. Freeman, E. Pomjakushina, K. Conder, and A. T. Boothroyd, Phys. Rev. B 83, 180506(R) (2011).

[34] K. Prokeš, A. Hiess, W. Bao, E. Wheeler, S. Landsgesell, and D. N. Argyriou, Phys. Rev. B 86, 064503 (2012).

[35] See supplementary information for details on how to estimate $M_{110}, M_{1 \overline{1} 0}$, and $M_{001}$.

[36] X. Y. Lu et al., unpublished. 


\section{Supplementary Materials}

Figure S1 shows the raw data of neutron SF scattering cross section $\sigma_{x, y, z}^{\mathrm{SF}}$ and the magnetic responses $M_{y}$ and $M_{z}$ at $E=3 \mathrm{meV}$ for three measured wave vectors $\mathbf{Q}_{\mathbf{1 , 2 , 3}}=(0.5,0.5,1),(0.5,0.5,3),(0.5,0.5,5)$, respectively. To deduce the three components of the magnetic excitations $M_{110}, M_{1 \overline{1} 0}$ and $M_{001}$, which correspond to spin excitations along the $a_{o}, b_{o}$, and $c$-axes of the orthorhombic lattice unit cell, we need to consider the angle dependence of spin excitations with respect to the neutron polarization directions [Fig. 1(g)]. For each measured wave vector $\mathbf{Q}_{\mathbf{1 , 2}, \mathbf{3}}$, we can estimate $M_{y}$ and $M_{z}$ from the raw data via

$$
\left\{\begin{array}{l}
\sigma_{x}^{\mathrm{SF}}-\sigma_{y}^{\mathrm{SF}}=c M_{y} \\
\sigma_{x}^{\mathrm{SF}}-\sigma_{z}^{\mathrm{SF}}=c M_{z}
\end{array}\right.
$$

where $c=(R-1) /(R+1)$ and the spin flipping ratio $R \approx 15$. The components of the magnetic responses along each of the crystallographic high symmetry directions can be written as: $M_{y}=M_{110} \sin ^{2} \theta+M_{001} \cos ^{2} \theta$ and $M_{z}=M_{1 \overline{1} 0}$ for each of the wave vectors, where $\theta$ is the angle between the neutron polarization direction $x$ and the $[1,1,0]$ direction as shown in Fig. $1(\mathrm{~g})$. These results are shown in Figs. S1(d) -(f) for wave vector $\mathbf{Q}_{\mathbf{1 , 2 , 3}}$.

In our experiments, the lattice parameters are $a \approx b \approx$ $3.956 \AA$, and $c=12.92 \AA$ using the pseudo-tetragonal structure, thus the angle $\theta$ between the wave vector $\mathbf{Q}=$ $[0.5,0.5, L]$ and the $[H, H, 0]$ direction can be calculated by using $\tan \theta=(2 \pi L / c) /\left(2 \pi \sqrt{(1 / 2 a)^{2}+(1 / 2 b)^{2}}\right)=$ $\sqrt{2} a L / c$, giving the results: $\theta_{1}=23.4^{\circ}, \theta_{2}=52.4^{\circ}$, $\theta_{3}=65.2^{\circ}$ for $\mathbf{Q}_{\mathbf{1 , 2 , 3}}=(0.5,0.5,1),(0.5,0.5,3),(0.5$, $0.5,5)$, respectively. Hence we have a series equations for $M_{y}$ from the three probed wave vectors:

$$
\left\{\begin{array}{l}
M_{y}\left(\mathbf{Q}_{\mathbf{1}}\right)=M_{110} \sin ^{2} 23.4^{\circ}+M_{001} \cos ^{2} 23.4^{\circ}=0.16 M_{110}+0.84 M_{001}, \\
M_{y}\left(\mathbf{Q}_{\mathbf{2}}\right)=M_{110} \sin ^{2} 52.4^{\circ}+M_{001} \cos ^{2} 52.4^{\circ}=0.63 M_{110}+0.37 M_{001}, \\
M_{y}\left(\mathbf{Q}_{\mathbf{3}}\right)=M_{110} \sin ^{2} 65.2^{\circ}+M_{001} \cos ^{2} 65.2^{\circ}=0.82 M_{110}+0.18 M_{001} .
\end{array}\right.
$$

Since $M_{110}$ and $M_{001}$ should be the same at these wave vectors except for the differences in the magnetic form factor and instrumental resolution, one can in principle unambiguously solve $M_{110}$ and $M_{001}$ if measurements at two equivalent wave vectors are carried out. As we can see, $M_{y}$ measurements at low wave vector $\mathbf{Q}_{\mathbf{1}}$ will be more sensitive to the $c$-axis polarized spin excitations $M_{001}$, while identical measurements at $\mathbf{Q}_{\mathbf{3}}$ will be more sensitive to $M_{110}$. Assuming that spin excitations in the system follow the $\mathrm{Fe}^{2+}$ magnetic form factor, we would expect that $F^{2}\left(\mathbf{Q}_{\mathbf{1}}\right)=0.826, F^{2}\left(\mathbf{Q}_{\mathbf{2}}\right)=0.652$, and $F^{2}\left(\mathbf{Q}_{\mathbf{3}}\right)=0.418$ for $\mathbf{Q}_{\mathbf{1 , 2}, \mathbf{3}}=(0.5,0.5,1),(0.5,0.5$, $3),(0.5,0.5,5)$, respectively. To estimate the contributions of instrumental resolution at different wave vectors, we note that instrumental contributions for spin excitations should be independent of neutron spin polarizations. If we assume that spin excitations $M 1 \overline{1} 0$ are identical for different wave vectors except for the magnetic form factor and instrumental resolution, we should have $M_{1 \overline{1} 0}=M_{z}\left(\mathbf{Q}_{\mathbf{1}}\right) /\left[F^{2}\left(\mathbf{Q}_{\mathbf{1}}\right) R_{1}\right]=M_{z}\left(\mathbf{Q}_{\mathbf{2}}\right) /\left[F^{2}\left(\mathbf{Q}_{\mathbf{2}}\right) R_{2}\right]=$ $M_{z}\left(\mathbf{Q}_{\mathbf{3}}\right) /\left[F^{2}\left(\mathbf{Q}_{\mathbf{3}}\right) R_{3}\right]$, where $R_{1}, R_{2}$, and $R_{3}$ are the scale factors representing contributions from instrumental resolutions at these wave vectors. Similarly, we have

$$
\left\{\begin{array}{l}
M_{y}\left(\mathbf{Q}_{\mathbf{1}}\right) /\left[F^{2}\left(\mathbf{Q}_{\mathbf{1}}\right) R_{1}\right]=0.16 M_{110}+0.84 M_{001}, \\
M_{y}\left(\mathbf{Q}_{\mathbf{2}}\right) /\left[F^{2}\left(\mathbf{Q}_{\mathbf{2}}\right) R_{2}\right]=0.63 M_{110}+0.37 M_{001}, \\
M_{y}\left(\mathbf{Q}_{\mathbf{3}}\right) /\left[F^{2}\left(\mathbf{Q}_{\mathbf{3}}\right) R_{3}\right]=0.82 M_{110}+0.18 M_{001}
\end{array}\right.
$$

Since we have measured SF scattering at three different wave vectors, we have over-determined the values of $M_{y}$ and $M_{z}$. Of the combined scale factors $F^{2}\left(\mathbf{Q}_{\mathbf{1}}\right) R_{1}$, $F^{2}\left(\mathbf{Q}_{\mathbf{2}}\right) R_{2}$, and $F^{2}\left(\mathbf{Q}_{\mathbf{3}}\right) R_{3}$, only two are independent as we do not measure $M_{1 \overline{1} 0}$ and $M_{110}$ in absolute units. Therefore, we can accurately determine $F^{2}\left(\mathbf{Q}_{\mathbf{2}}\right) R_{2}$ and $F^{2}\left(\mathbf{Q}_{\mathbf{3}}\right) R_{3}$ (assuming $F^{2}\left(\mathbf{Q}_{\mathbf{1}}\right) R_{1}=1$ ) using measured values of $M_{y}$ and $M_{z}$. We do not need to know the values of the magnetic form factor. This procedure will also allow us to unambiguously determine the temperature dependence of $M_{110}, M_{1 \overline{1} 0}$, and $M_{001}$ as shown in Fig. $5(\mathrm{~d})$. In any case, Figs. S1(e) and S1(f) show clear differences between $M_{y}$ and $M_{z}$ at $35 \mathrm{~K}$. Since at $M_{y}\left(\mathbf{Q}_{\mathbf{3}}\right)$ has $82 \%$ contribution from $M_{110}$ and $M_{z}$ is $100 \% M_{1 \overline{1} 0}$, inplane spin excitations are unambiguously anisotropic in the paramagnetic tetragonal state of $\mathrm{BaFe}_{1.904} \mathrm{Ni}_{0.096} \mathrm{As}_{2}$ without an uniaxial pressure. 


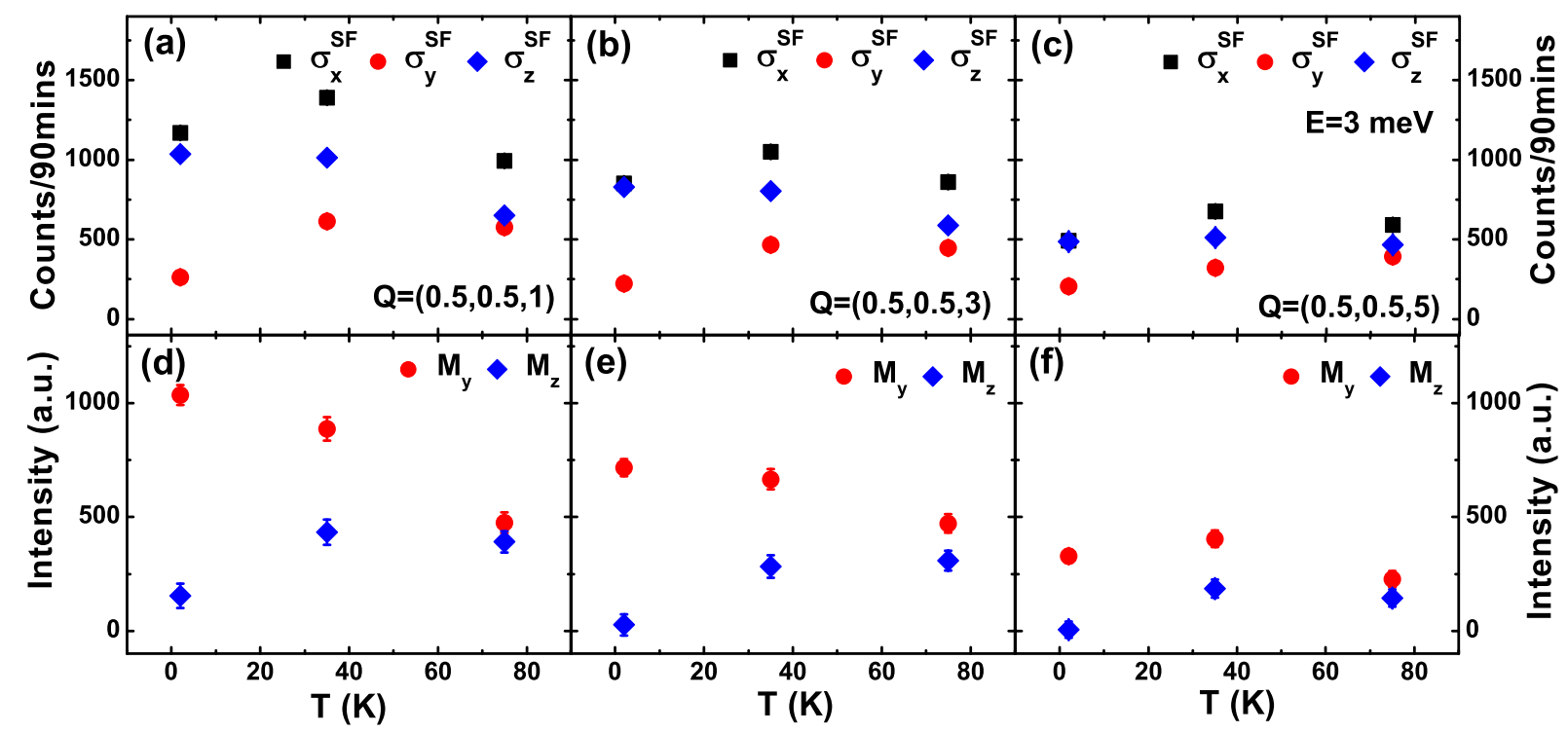

FIG. S1: (a)-(c) Temperature dependence of neutron SF scattering cross section $\sigma_{x, y, z}^{\mathrm{SF}}$ at $E=3 \mathrm{meV}$ for $\mathbf{Q}_{\mathbf{1 , 2 , 3}}=$ $(0.5,0.5,1),(0.5,0.5,3),(0.5,0.5,5)$ in the scattering geometry shown in Fig. $1(\mathrm{~g})$. (d)-(f) The magnetic response $M_{y}$ and $M_{z}$ at the three wave vectors. 